\title{
On the Evolution of Hexose Transporters in Kinetoplastid Potozoans
}

\author{
Claudio Alejandro Pereira ${ }^{1 * 9}$, Ariel Mariano Silber ${ }^{2 * 9}$
}

1 Instituto de Investigaciones Médicas "Alfredo Lanari," Ciudad Autónoma de Buenos Aires, Argentina, 2 Departamento de Parasitologia, Instituto de Ciências Biomédicas, Universidade de São Paulo, São Paulo, São Paulo, Brasil

\begin{abstract}
Glucose, an almost universally used energy and carbon source, is processed through several well-known metabolic pathways, primarily glycolysis. Glucose uptake is considered to be the first step in glycolysis. In kinetoplastids, a protozoan group that includes relevant human pathogens, the importance of glucose uptake in different phases of the life cycles is well established, and hexose transporters have been proposed as targets for therapeutic drugs. However, little is known about the evolutionary history of these hexose transporters. Hexose transporters contain an intracellular N- and C- termini, and 12 transmembrane spans connected by alternate intracellular and extracellular loops. In the present work we tested the hypothesis that the evolutionary rate of the transmembrane span is different from that of the whole sequence and that it is possible to define evolutionary units inside the sequence. The phylogeny of whole molecules was compared to that of their transmembrane spans and the loops connecting the transmembrane spans. We show that the evolutionary units in these proteins primarily consist of clustered rather than individual transmembrane spans. These analyses demonstrate that there are evolutionary constraints on the organization of these proteins; more specifically, the order of the transmembrane spans along the protein is highly conserved. Finally, we defined a signature sequence for the identification of kinetoplastid hexose transporters.
\end{abstract}

Citation: Pereira CA, Silber AM (2012) On the Evolution of Hexose Transporters in Kinetoplastid Potozoans. PLoS ONE 7(5): e36303. doi:10.1371/ journal.pone.0036303

Editor: Fabio T. M. Costa, State University of Campinas, Brazil

Received August 30, 2011; Accepted April 2, 2012; Published May 2, 2012

Copyright: (c) 2012 Pereira, Silber. This is an open-access article distributed under the terms of the Creative Commons Attribution License, which permits unrestricted use, distribution, and reproduction in any medium, provided the original author and source are credited.

Funding: This work was supported by grants from the Consejo Nacional de Investigaciones Científicas y Técnicas (CONICET, PIP Grant \#0685 to CAP), and Agencia Nacional de Promoción Científica y Tecnológica (FONCYT PICT Grant \#2008-1209 to CAP), Fundação de Amparo à Pesquisa do Estado de São Paulo (FAPESP grants \#11/50631-1 to AMS) and Instituto Nacional de Biologia Estrutural e Química Medicinal em Doenças Infecciosas (INBEQMeDI). CAP is a member of the career of scientific investigator of CONICET (Argentina). The funders had no role in study design, data collection and analysis, decision to publish, or preparation of the manuscript.

Competing Interests: The authors have declared that no competing interests exist.

*E-mail: cpereira@retina.ar (CAP); asilber@usp.br (AMS)

9 These authors contributed equally to this work.

\section{Introduction}

The order Kinetoplastidae consists of flagellated protozoans that have a peculiar mitochondrial DNA-containing structure called kinetoplast. This group includes species of parasitic protozoa of the genera Trypanosoma and Leishmania, which cause severe human disease. Two species of the genus Trypanosoma, T. brucei and $T$. cruzi, are the causative agents of sleeping sickness and Chagas' disease, respectively. Species of the Leishmania subgenera Leishmania and Viannia are the causative agents of a group of diseases known together as leishmaniasis. Together, these parasites affect approximately 25 million people in endemic areas all over the world, with an estimated population of more than 350 million people at risk of acquiring these infections. Unfortunately, the drugs used to treat human infections by these parasites are unsatisfactory: their low therapeutic efficiency, high toxicity, and the appearance of resistant strains constitute serious drawbacks that remain to be overcome [1].

Kinetoplastid protozoa usually have a complex life cycle, alternating between one or more hosts and frequently moving between several territories with different nutrient compositions inside their hosts (i.e., different regions of the insect midgut). The ability of these cells to deal with these environmental changes is reflected by their flexible metabolism, which allows them to use glucose or amino acids (mainly proline) as their main carbon and energy source $[2,3]$. The cells usually consume amino acids only when they are subjected to glucose deprivation conditions $[4,5]$, supporting the idea that glucose consumption is the default option and emphasizing the relevance of this carbohydrate for these organisms [6].

Transporters are essential proteins in most cells and are among the first molecules involved in the detection of their substrates in the extracellular medium [7]. In trypanosomatids, glucose uptake can be considered to be the first regulated step of glycolysis [8]. These findings led to the early study of glucose uptake pathways in pathogenic trypanosomatids and to molecular studies of hexose transporters in T. brucei, T. cruzi and Leishmania spp. parasites [9]. The relevance of glucose metabolism has been particularly well demonstrated in these trypanosomatids, and hexose transporters and several stages of glycolysis have been proposed as drug targets $[10,11]$. More recently, with the advance of the T. brucei, T. cruzi and L. major (TriTryp) genome projects, additional hexose transporter genes belonging to other pathogenic and nonpathogenic kinetoplastids have been annotated [12], and some of them have been experimentally validated [8]. All of the glucose transporters described to date in kinetoplastids are related to solute 
carrier family 2, also known as facilitated glucose transporter member 1 (SLC2A1) or GLUT1, belonging to the Major Facilitator Superfamily (2.A.1, MFS). Although we have a considerable amount of information about the GLUT1 family, little is known about the evolution of these peculiar proteins [13].

GLUT1 is a glucose transporter family that is broadly distributed across Eukarya and Bacteria [14]. The structure of these facilitative hexose permeases is supported exclusively by twelve transmembrane spans, which are connected by extracellular and intracellular loops $[15,16]$. Because the transport activity of these proteins relies on their transmembrane regions, we hypothesize here that the connecting loops have fewer constraints with regard to amino acid substitution during evolution. Following this rationale, we hypothesize that the transmembrane regions of hexose transporters constitute evolutionary modules with more constrained variation patterns than the extracellular and intracellular loops. In the present work we used the kinetoplastid hexose transporter sequences available in the TriTryp database [17] to analyze the evolutionary history of these proteins in species belonging to the genera Trypanosoma and Leishmania, with particular focus on the transmembrane regions. The results presented herein show for the first time that clusters of consecutive transmembrane spans constitute evolutionary modules.

\section{Materials and Methods}

\section{Sequence retrieval and domain identification}

All available amino acid sequences annotated as hexose/glucose transporters were recovered from the TriTryp database version 3.2 (http://tritrypdb.org/tritrypdb) [17]. The species and accession numbers of the sequences used are listed in Table 1. Only sequences corresponding to a single allelic copy per species were chosen to be included in the present analysis. The initial search for constructing the starting-up database was performed as follow. Due to the presence of two haplotypes (Esmeraldo and nonEsmeraldo) in the available genome data from the T. cruzi CL Brener strain, only one representative gene was analyzed; T. cruzi Esmeraldo if it exists, otherwise T. cruzi non-Esmeraldo if it exists, otherwise $T$. cruzi unassigned genes. In the case of T. brucei, nonredundant genes were also analyzed considering the representative gene this corresponding to T. brucei strain TREU927 if it exists, otherwise T.brucei strain 427 if it exists, otherwise T. brucei gambiense. Sequences from other Trypanosoma spp. and Leishmania spp. were included if their identity were less than 98\%. The obtained sequences were curated initially on the basis of their similarities to glucose transporters by using the online version of BLASTp at the NCBI (http://www.ncbi.nlm.nih.gov/BLAST/). BLASTp was run under default parameters using the non-redundant protein sequence database. The sequences were further analyzed for the position of the 12 transmembrane spans with SOSUI v1.11 [18], TMHMM v2.0 [19], TMPRED v1.0 (http://www.ch.embnet. org/software/TMPRED_form.html) and HMMTOP v2.0 (http://www.enzim.hu/hmmtop/). The predicted transmembrane regions were recovered as independent sequences for further analysis. Assemblies and analysis of the amino acid sequence data were carried out using the software package Vector NTI v. 10.3.0 (Invitrogen, California - USA).

\section{Phylogenetic and other bioinformatic analyses}

Phylogenetic analyses were performed using Molecular Evolutionary Genetics Analysis (MEGA) v5.05 [20,21,22]. Briefly, the evolutionary history was inferred with the maximum likelihood method with a JTT matrix-based model. The bootstrap consensus tree inferred from 500 replicates was taken to represent the
Table 1. Description of the main characteristics of the used sequences.

\begin{tabular}{|c|c|c|c|c|c|c|}
\hline AN & Species & Length & TMS & $\begin{array}{l}\text { NTP/ } \\
\text { CTP }\end{array}$ & NTL & CTL \\
\hline LbrM.33.0290 & L. braziliensis & 617 & 12 & $\mathrm{I} / \mathrm{I}$ & 94 & 27 \\
\hline LbrM.35.6490 & L. braziliensis & 531 & 12 & $\mathrm{I} / \mathrm{I}$ & 20 & 24 \\
\hline LinJ.36.6550 & L. infantum & 567 & 12 & $\mathrm{I} / \mathrm{I}$ & 46 & 32 \\
\hline LinJ.36.6560 & L. infantum & 653 & 12 & $\mathrm{I} / \mathrm{I}$ & 135 & 32 \\
\hline LmjF.33.0290 & L. major & 594 & 12 & $\mathrm{I} / \mathrm{I}$ & 73 & 24 \\
\hline LmjF.36.6280 & L. major & 568 & 12 & $\mathrm{I} / \mathrm{I}$ & 46 & 32 \\
\hline LmjF.36.6290 & L. major & 561 & 12 & $1 / 1$ & 45 & 26 \\
\hline LmjF.36.6300 & L. major & 653 & 12 & $\mathrm{I} / \mathrm{I}$ & 135 & 32 \\
\hline LmxM.32.0290 & L. mexicana & 627 & 12 & $\mathrm{I} / \mathrm{I}$ & 107 & 24 \\
\hline LmxM.36.6280 & L. mexicana & 566 & 12 & $\mathrm{I} / \mathrm{I}$ & 48 & 32 \\
\hline LmxM.36.6290 & L. mexicana & 567 & 12 & $\mathrm{I} / \mathrm{I}$ & 49 & 32 \\
\hline LmxM.36.6300 & L. mexicana & 610 & 12 & $1 / 1$ & 88 & 32 \\
\hline Tb427.04.2290 & T. brucei & 552 & 12 & $\mathrm{I} / \mathrm{I}$ & 51 & 07 \\
\hline Tb427.10.8450 & T. brucei & 527 & 12 & $\mathrm{I} / \mathrm{I}$ & 43 & 30 \\
\hline Tb427.10.8530 & T. brucei & 529 & 12 & $\mathrm{I} / \mathrm{I}$ & 41 & 31 \\
\hline Tc00.1047053506355.10 & T. cruzi & 544 & 12 & $\mathrm{I} / \mathrm{I}$ & 34 & 47 \\
\hline TcIL3000.10.7320 & $\begin{array}{l}T . \\
\text { congolense }\end{array}$ & 525 & 12 & $1 / 1$ & 40 & 29 \\
\hline TvY486_0402140 & T. vivax & 558 & 12 & $1 / 1$ & 48 & 09 \\
\hline Ecoli_P02920 (Lac Y) & E. coli & 417 & 12 & I/I & 07 & 19 \\
\hline
\end{tabular}

AN: Accession Numbers; TMS: number of transmembrane spans in each sequence; NTP: N-terminal position; CTP: C- terminal position; NTL: Nterminal length; I: intracellular; E: extracellular.

doi:10.1371/journal.pone.0036303.t001

evolutionary history of the sequences analyzed. Branches corresponding to partitions reproduced in fewer than $50 \%$ of bootstrap replicates were collapsed. Initial tree(s) for the heuristic search were obtained automatically as follows. When the number of common sites was lower than 100 or less than one-fourth of the total number of sites, the maximum parsimony method was used; otherwise, the BIONJ method with the MCL distance matrix was used. The trees were drawn to scale, with branch lengths measured in the number of substitutions per site. All positions containing gaps and missing data were eliminated. The average evolutionary divergence over all sequence pairs was also calculated using the JTT matrix-based model involving all amino acid sequences in each case. Similarity analyses based on the consensus sequence were conducted using the ClustalW algorithm [23]. For the identification of possible specific signatures, all sequences were scanned using Multiple Em for Motif Elicitation (MEME) v4.6.1 $[24]$.

\section{Results}

Our search for all amino acid sequences annotated as hexose or glucose transporters in the TriTryp database returned 57 sequences (Table 1). To eliminate redundancies all allelic copies $(>98 \%$ identity), truncated sequences and pseudogenes were discarded. Of the remaining 18 sequences distributed among 7 species of the genera Trypanosoma and Leishmania, the functions of 10 had been experimentally verified, while the functions of the other 8 had been inferred from sequence data $[9,25,26,27,28,29,30,31]$. 
Lbra contig Larm 35

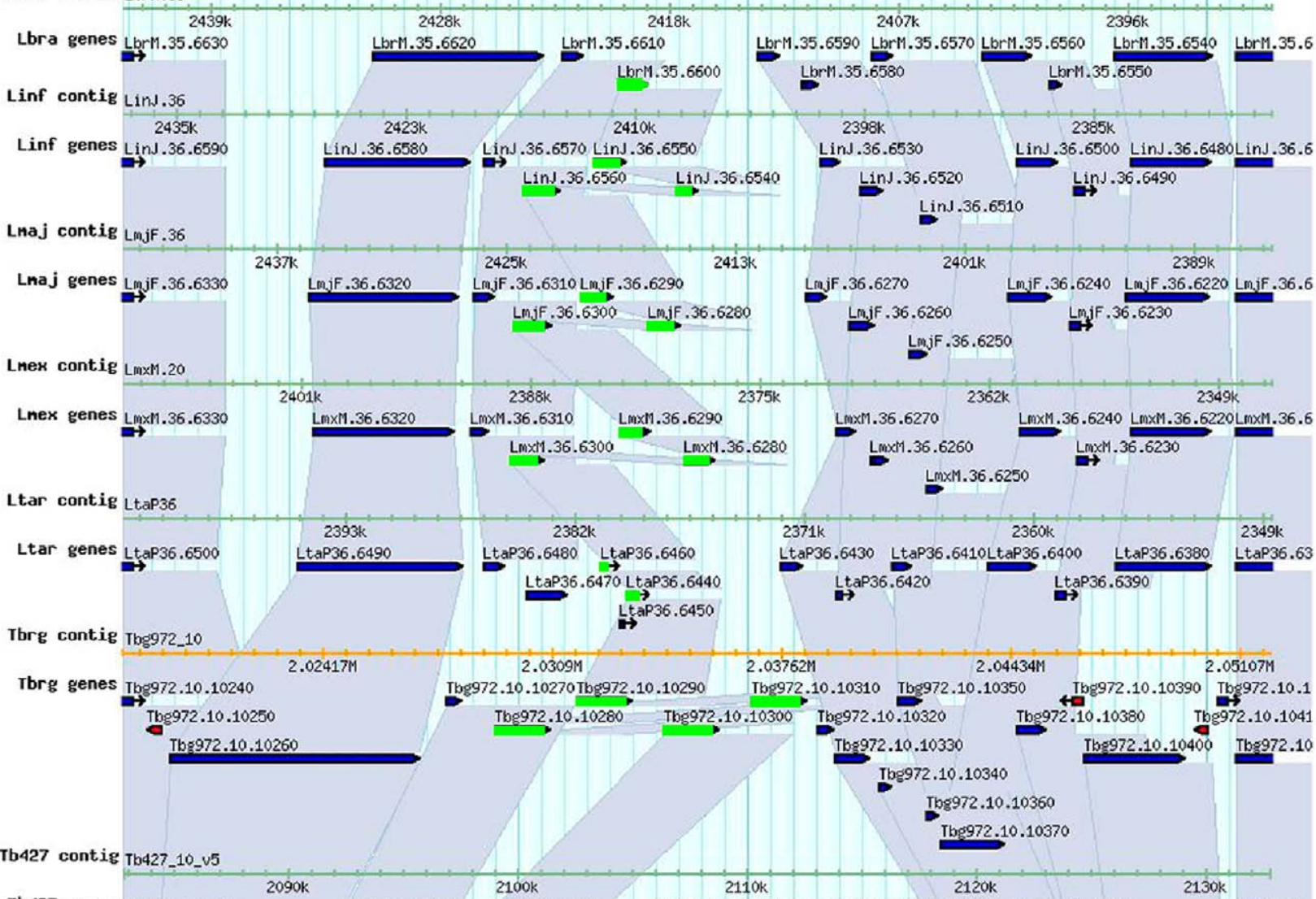

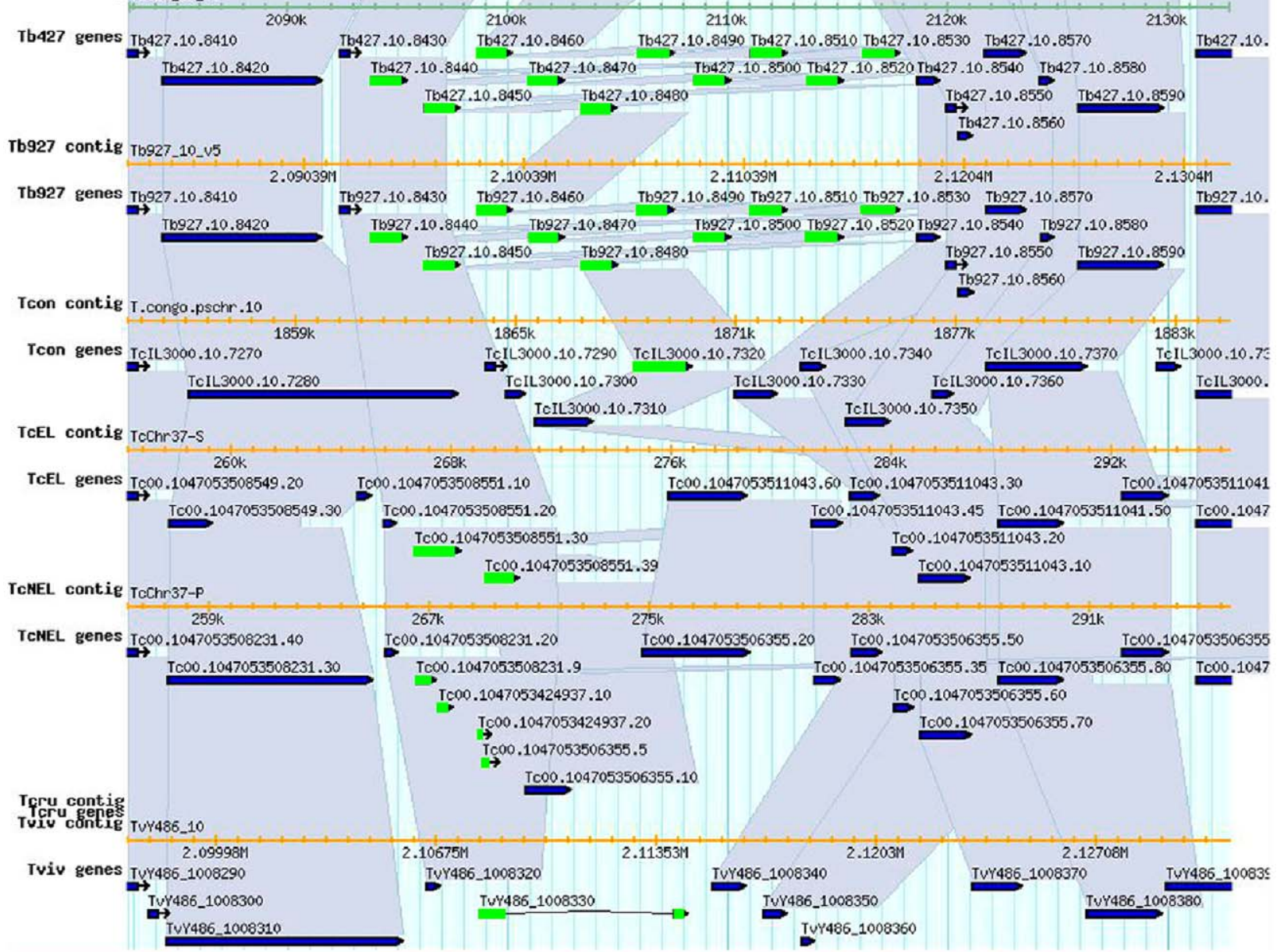


Figure 1. Hexose transporters synteny analysis. The genomic contexts of the Trypanosoma spp and Leishmania spp hexose transporters were analyzed. The figure represent one syntenic orthologous group (highlighted in green) comprising the genes LinJ.36.6550 (and its paralog LinJ.36.6560), LmjF.36.6280 (and its paralogs LmjF.36.6290-LmjF.36.6300, called Imgt1-3), LmxM.36.6280 (and its paralogs LmxM.36.6290LmxM.36.6300), TcIL3000.10.7320, and Tb427.10.8450 (and its paralog Tb427.10.8530, called THT1). This group of genes has expanded into 10 tandem duplications. Lila shadows indicates the syntenic genes and all the sequences are represented by their systematic ID. The synteny analyses and scheme was made at the TriTrypDB (http://tritrypdb.org/tritrypdb). doi:10.1371/journal.pone.0036303.g001

The analyzed genes fall into three syntenic orthologous groups. The first group comprises the genes LinJ.36.6550 (and its paralog LinJ.36.6560), LmjF.36.6280 (and its paralogs LmjF.36.6290LmjF.36.6300, called lmgt1-3), LmxM.36.6280 (and its paralogs LmxM.36.6290-LmxM.36.6300), TcIL3000.10.7320 and Tb427.10.8450 (and its paralog Tb427.10.8530, called THT1). This group of genes has expanded into 10 tandem duplications (Figure 1). The second group comprises the orthologs LbrM.33.0290, LmjF.33.0290 and LmxM.32.0290, and the third group comprises Tb427.04.2290 and TVY486_0402140. LbrM.35.6490 and Tc00.1047053506355.10 have no orthologs.

All of these sequences were analyzed for the presence of putative transmembrane regions. To make the analysis more robust, four different algorithms were used to define the most probable transmembrane regions and the orientation of the $\mathrm{N}$ - terminal region of each sequence. As previously mentioned, all of the hexose transporter sequences analyzed in the present study belong to the major facilitator superfamily (MFS) [32].

Because the lactose permease (LacY) from Escherichia coli is a well-known member of this superfamily and its structure has been extensively studied $[33,34]$, we used it as a model for inferring the structural aspects of trypanosomatid transporters. In all cases, 12 transmembrane domains could be detected (Table 1). These domains were then excised from the surrounding sequence for further analysis. In this way, we obtained 216 fragments, which were subsequently treated as separate sequences (Dataset S1).

To infer the evolutionary history of trypanosomatids hexose transporters, we submitted their amino acid sequences to phylogenetic analysis by the maximum likelihood method using 500 bootstrap replicates (Figure 2A). To root the trees, sequences corresponding to the hexose transporter family GLUT1 from several unrelated organisms, that is, Homo sapiens, Drosophila melanogaster, Danio rerio and Escherichia coli Lac Y, were used. Among the trypanosomatid sequences, we observed that two sequences, Tb427.04.2290 and TVY486_0402140, branched earlier than the rest. The remaining 16 sequences were mainly distributed into two branches: one branch grouped the only $\mathcal{T}$. vivax sequence with one out of three $T$. brucei sequences, while the other branch contained all other transporters. This second branch was divided into two main clusters, which corresponded to the genera Leishmania and Trypanosoma.

Hexose transporters, similar to other integral membrane proteins, are divided into defined modules, i.e., the transmembrane regions, which are essential for protein activity. Consequently, we hypothesized that the evolution of the transmembrane domains should be more constrained than that of the whole molecule. To test this hypothesis, for each full-length protein, we generated the following: 1) a sequence in which we removed the intracellular and extracellular loops connecting the transmembrane regions (loops or L), and 2) a sequence in which the transmembrane spans (TMS) were removed. Thus, for each fulllength original sequence, we obtained one sequence containing only the transmembrane spans (transmembrane spans joined, or TMSJ) and one sequence containing only the loops connecting the transmembrane regions (loops joined, or LJ) (Figure 2B). These sequences were then resubmitted to phylogenetic analysis by the maximum likelihood method, again using 500 bootstrap replicates (Figures $3 \mathrm{~A}$ and $\mathrm{B}$ ). The resulting trees were similar to those obtained when the full-length protein sequences were used, showing two defined clusters that separated the sequences by genus. This finding suggests that, in qualitative terms, the evolution of the transmembrane regions did not differ from that of the full-length sequence. We also analyzed the mean similarity among the full-length, TMSJ and LJ sequences. Percent similarity values of $73 \%$ and $80 \%$ were calculated for the full-length and TMSJ sequences, respectively, while the values obtained for the variable $\mathrm{N}$ - termini and the $\mathrm{LJ}$ sequences were $12 \%$ and $49 \%$, respectively (Figure 3C). Taken together, these data show that the transmembrane regions of these sequences are more stable over evolutionary time than the whole molecule, the $\mathrm{N}$ - terminus or the LJ. Thus, these results support the idea that the transmembrane domains of hexose transporters are the most evolutionarily constrained modules in these molecules.

From the data obtained, two models can be proposed for the evolution of hexose transporters in trypanosomatids: 1. The proteins evolved as a whole from a full-length ancestor, or 2. The proteins evolved through the independent evolution of the constituent modules. To distinguish between these models, the transmembrane span regions (TMS) or the N- and C- termini together with the loops connecting the transmembrane regions (loops, or L) of all sequences were extracted and numbered consecutively (based on the position they occupy in the original sequence). Thus, the sequences designated as "l" were located closest to the $\mathrm{N}$ - termini (Figure 2B). For this work, all 216 transmembrane regions and 234 loops were treated individually as single evolutionary units and were analyzed by the maximum likelihood method. TMSs from the sequences used as outgroups, including LacY, were also analyzed. Interestingly, we observed that most of the TMS sequences segregated into two major groups, which branched close to the base of the tree (see Figure 4A). The consecutively numbered spans formed two coherent superclusters organized as follows: A) 1, 4-7, and B) 2-3, 8-11 and 12 (Figure 4A).

The same method was used to analyze the loops connecting the transmembrane regions. Unlike what occurred with the TMS sequences, we observed that the loop sequences segregated into randomly distributed clusters (Figure S1). Due to the particularly high levels of divergence in these sequences, the use of distance estimation analysis by overall mean distances (based on the JTT algorithm) failed in detecting common sites among the sequences. Taken together, these results support the idea that these molecules have modules of evolution, consisting of single transmembrane regions or discrete groups of transmembrane regions.

We next sought to examine the evolution of the order in which these modules are arranged. To approach this problem, we first made identity plots for sequences in which the transmembrane spans were joined in sequential order (TMSJ) or randomly scrambled (S-TMSJ, see Figure 2B). We observed high levels of identity inside each transmembrane span and low levels in the interface regions (the position at which one span ends and the next one begins). However, when the order of the transmembrane regions was scrambled, we observed that identities as a function of 


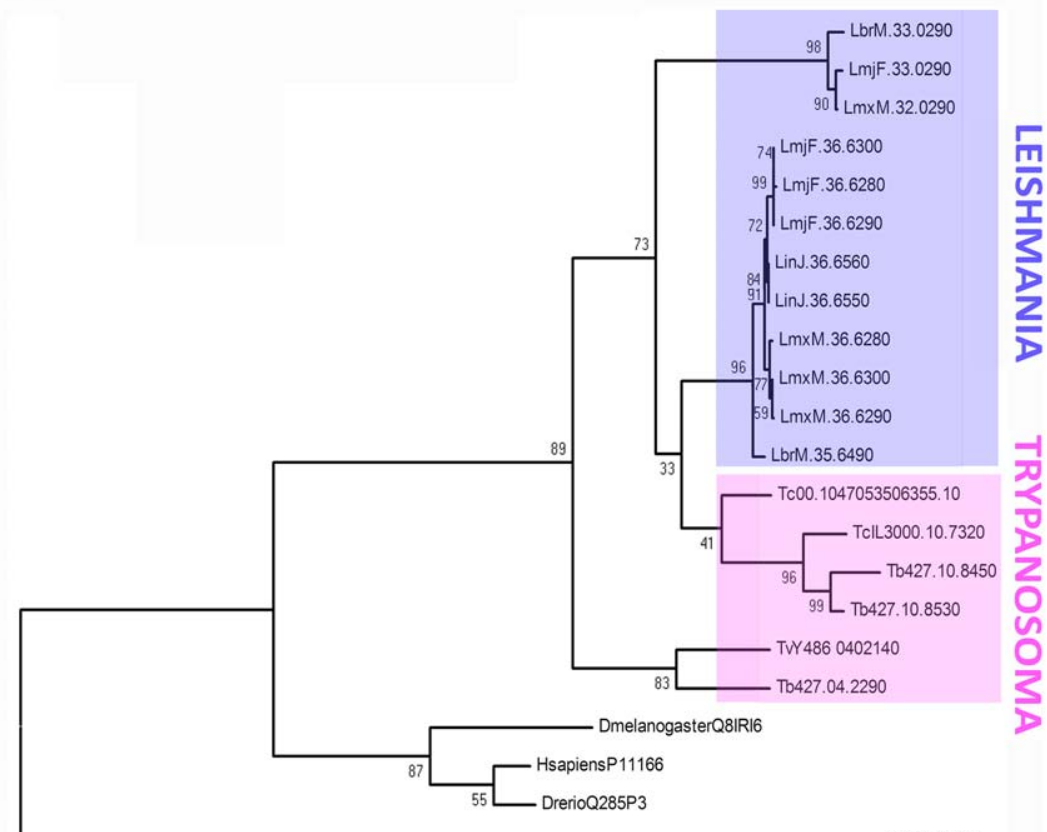

0.5

B

Full-length sequence (FL)

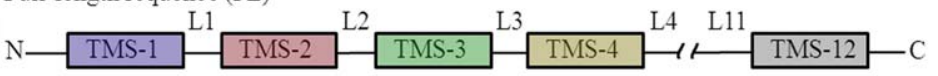

Trans-membrane spanners joined (TMSJ)

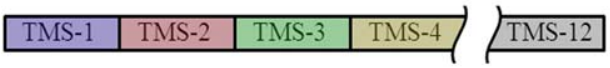

Scrambled trans-membrane spanners joined (S-TMSJ)

\begin{tabular}{|l|l|l|l|}
\hline TMS-3 & TMS-5 & TMS-6 & TMS-11 \\
\hline TMS-2 \\
\hline
\end{tabular}

Loops joined (LJ)

Scrambled loops joined (S-LJ)

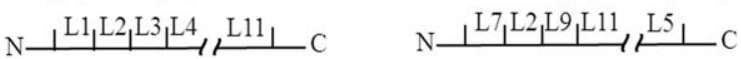

Figure 2. Phylogeny of the hexosetransporters. A: Evolutionary tree constructed using the full-length amino acid sequences of the kinetoplastid hexose transporters. Sequences coding for glucose transporters of Homo sapiens, Drosophila melanogaster, Danio rerio and Escherichia coli were used as outgroups to root the tree. A maximum likelihood method based on the JTT matrix-based model was used, with 500 bootstrap replicates. The tree with the highest log likelihood $(-7654.7770)$ is shown. The tree is drawn to scale, with branch lengths measured in the number of substitutions per site. The kinetoplastid hexose transporters segregate into two main groups, which correspond to the genera Trypanosoma and Leishmania. B: Schematic representation of the structure of the sequences used. FL: full-length sequence, TMS: transmembrane span, L: loops connecting the TMSs, TMSJ: sequence containing contiguously ordered TMSs, LJ: sequence containing contiguously ordered Ls, S-TMSJ: scrambled TMSJ, S-LJ: scrambled SJ.

doi:10.1371/journal.pone.0036303.g002

amino acid position were completely lost: the mean global identity values diminished from $71.9 \%$ for TMSJ to $20.4 \%$ for S-TMSJ. When the same analysis was performed on the connecting loops, the identity values decreased for both the LJ and S-LJ sequences (36.5 and $12.2 \%$, respectively) (Figure $4 \mathrm{~B}$ ), indicating a small contribution of the order of the $\mathrm{L}$ regions to global identity (see values in Figure 3C). This result strongly indicates that, in addition to the fact that individual or clusters of transmembrane regions act as evolutionary modules, their order inside the molecule is critical for functional hexose transporters. To estimate the evolutionary divergence between TMSs in a single sequence and between
TMSs at the same location in different sequences, the TMSs were grouped by constructing a matrix $\mathbf{M}[i, j]$, where i represents a sequential array of TMSs from the same molecule (TMS-1, ... TMS-12) and $\mathrm{j}$ represents TMS-i for each of the 18 sequences under analysis (TMS-1,1, .., TMS-1,18) (Table S1). The whole matrix divergence was calculated to be 7.7 arbitrary units. This value was similar to those obtained within each row and among rows and to that calculated among columns. Interestingly, the mean divergence within each column was 1.45; the column corresponding to TMS-1 was the only one with a high divergence value (8.46). Interestingly, the $\mathrm{N}$ - and $\mathrm{C}$ - termini were the most 
A

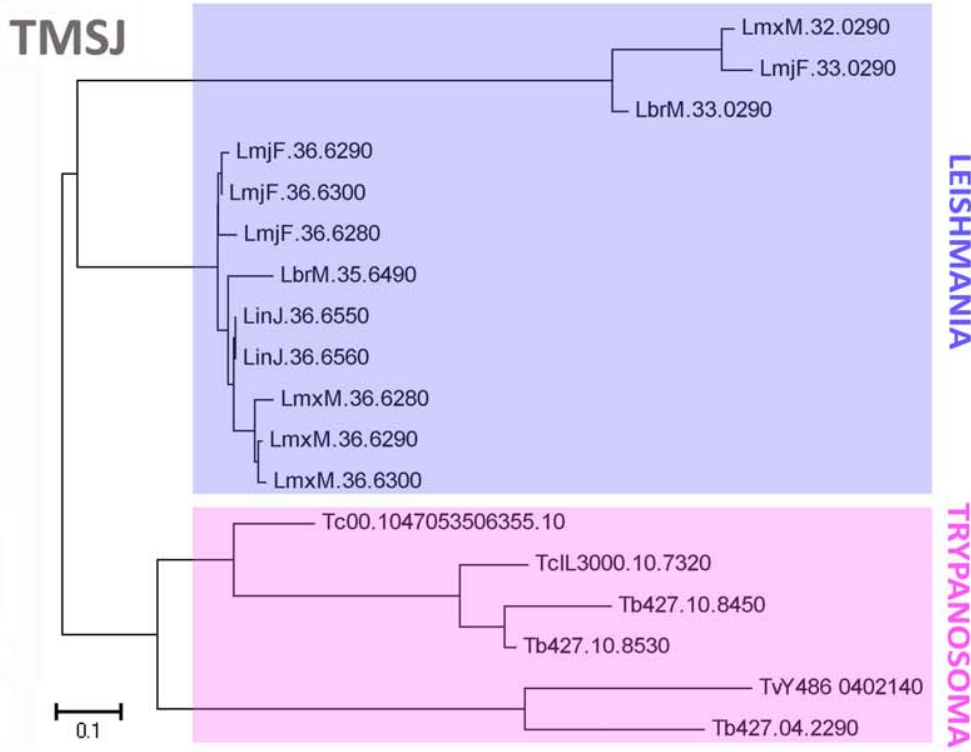

B

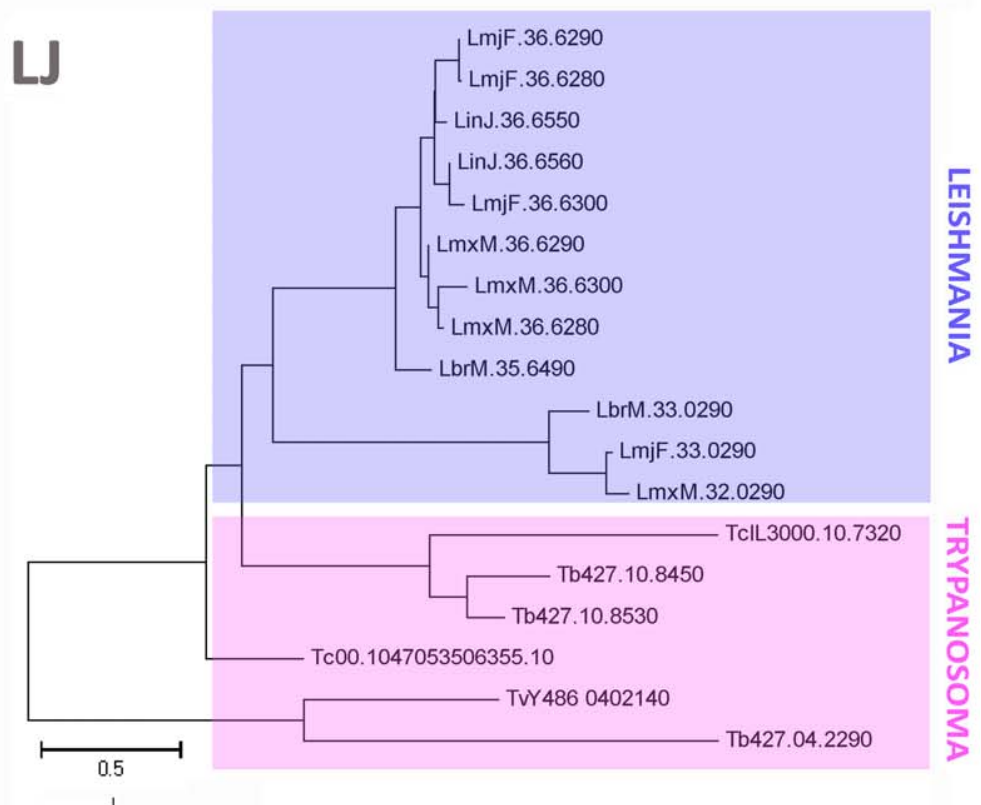

\begin{tabular}{|l|lc}
\hline \multicolumn{1}{|c|}{} & $\begin{array}{c}\text { SIMILARITY } \\
\text { (\%) }\end{array}$ \\
\hline FL & 73 \\
TMS & 19 \\
TMSJ & 80 \\
S-TMSJ & 23 \\
NT & 12 \\
U & 49 \\
S-LJ & 13 \\
\hline
\end{tabular}


Figure 3. Evolutionary trees of transmembrane spans and loops. Evolutionary tree using the TMSJ (A) or $L$ (B) sequences. A maximum likelihood method based on the JTT matrix-based model was used, with 500 bootstrap replicates. Trees with the highest log likelihood (-3067.0851 and -4129.5431 , respectively) are shown. Trees are drawn to scale, with branch lengths measured in the number of substitutions per site. In both cases, sequences segregate into two main groups corresponding to the genera Trypanosoma and Leishmania. C: The mean distances for each group of sequences analyzed (FL, TMS, TMSJ, S-TMSJ, NT, LJ and S-LJ) were calculated and are presented as similarity values. doi:10.1371/journal.pone.0036303.g003

divergent regions along the whole molecule. These data indicate that the TMSs corresponding to the same positions in different proteins are the most conserved evolutionary units of kinetoplastid hexose transporters. In addition, although it is likely that these proteins evolved by internal TMS duplications, no traces of this process remain detectable in the sequences under analysis. This conclusion is supported by our analysis of internal amino acid repeats in these proteins using four different algorithms, none of which found evidence of TMS duplications.

The presence of conserved regions in the hexose transporters studied could prove valuable for identifying critical regions related to the protein activity. All of the sequences were analyzed using the Multiple Em for Motif Elicitation (MEME) algorithm (http:// meme.nbcr.net/). This analysis identified the motif QLTGINA (which we refer to as the "GINA" motif), which was primarily present in TMS-6 and TMS-7 (Figure S2).

\section{Discussion}

The kinetoplastid hexose transporters have low but significant identity to members of the mammalian GLUT1 family [35]. For those hexose transporters that have been cloned and have had their activity demonstrated, it is predicted that the proteins contain 12 transmembrane spans [25,26,27,31,36,37,38,39,40,41]. However, this should not be taken as a rigid rule. As the genome projects for more trypanosomatids progress, new sequences are being annotated as putative hexose transporters, some of which have 10 or 11 transmembrane regions predicted by validated bioinformatic tools [12]. In the present study, we found 57 hexose transporters annotated for seven pathogenic and non-pathogenic kinetoplastid species (listed in Table 1). After curating the obtained sequences, we arrived at a total of 18 sequences, all them bearing 12 transmembrane spans. Because glucose transporters are essential for most living cells, new ones will likely be found in other trypanosomatid genomes. In this study, we detected a signature for kinetoplastid hexose transporters, the "GINA" motif (QLTGINA), which could be useful for detecting putative hexose transporters in newly sequenced kinetoplastids.

The probable structure of the kinetoplastids hexose transporters was classically based on that of the GLUT1 members of the GLUT family [9], which belong to the major facilitator superfamily (MFS) [13]. Most of members of this superfamily are predicted to contain 12 transmembrane helices based on the hydropathy plot for GLUT1 [13] which was first described by Mueckler et al. [42]. One of the best studied members of this group is the $E$. coli $\mathrm{LacY}$ protein, the structure of which was resolved in detail by crystallographic studies [33,34]. This protein consists of 12 transmembrane regions, which act as the structural support for the protein's transport activity. In the LacY crystallography model, it was established that the spans are not arranged in space in sequential order (i.e., helix 1 is surrounded by helices 4 and 5 instead of helix 2). LacY is organized into two groups of six helices each (I to VI and VII to XII), and both groups are connected by a long loop between helices VI and VII. These groups of $\mathrm{N}$ - and $\mathrm{C}$-terminal TMSs display two-fold symmetry. In addition, the $\mathrm{N}$ - and $\mathrm{C}$-terminal halves present some weak similarities, suggesting gene duplication or fusion. Based on the known structure of $\mathrm{LacY}$, we modeled the $\mathcal{T}$. cruzi hexose transporter, which is a well characterized and representative member of the others analyzed in the present study. Interestingly, the model presented reliable adjusting parameters, with an e-value of $1.9 \times 10^{-27}$ (Figure S3). Based on their structural similarity, we expected that evolutionary modules would correspond to neighboring TMSs, but this was not the case. This finding could be explained by the fact that, differently from what was predicted for human GLUT1, it was not expectable that trypanosomatid hexose transporters interact with other proteins: the only human GLUT1 known interactor (beside itself [43]) is GLUT1CBP [44], which is absent in trypanosomatids.

The $\mathrm{N}$ - terminus, TMS-1, L-1 and the C- terminus were generally the most divergent regions of GLUT proteins. In fact, the large extracellular loop between helices 1 and 2, present in all sequences, is one of the factors that diminished the quality of the LacY-based models. This result is not surprising because this feature, while common in the GLUT1 family, is absent in LacY [13]. When these regions were excluded, the resulting models showed that trypanosomatid transporters had a similar architecture to that of LacY (Figure S3). As discussed for Lac Y, two domains were present, constituted by helices 2 to 6 (corresponding to the N-terminal half of the molecule), and constituted by helices 7 to 12 (corresponding to the C-terminal half of the molecule). Based on the model, both halves seem to be linked by a long intracellular loop and surround a predicted cavity that opens to the cytoplasmic side of the membrane. It is also assumed that both the $\mathrm{N}$ - and C-termini are intracellular.

The differences in the role of transmembrane regions of most metabolite transporters, which are critical for their correct insertion into the membrane, as well as for their conformation and activity [7], led us to analyze if these TMSs should be more constrained during evolution than the loops which seem to be more prone to amino acid substitutions. Indeed, the only predictable evolutionary constraint for the loops would be related to the avoidance of highly immunogenic structures on the parasite's surface. The hexose transporters provide a very interesting and biologically relevant model to test the hypothesis that intramolecular evolutionary modules exist. Initially, we hypothesized that the TMSs would act as individual modules. However, the phylogenetic tree obtained with the TMSs showed a more complex pattern, including a positional clustering of consecutively ordered sequences (TMSs 1 and 2 or 11 and 12), strongly supporting the idea that these modules could originate via duplication. However, non-positional clustering (i.e., of TMSs that are not necessarily contiguous) was also observed. This finding could be explained by possible interactions between non-contiguous TMSs as reported for LacY.

The arrangement of the genes coding the trypanosomatids hexose transporters in their respective genomes was also analyzed. An interesting novelty is the fact that some trypanosomatids' transporters are expanded into multiple tandem duplications, and most of them are syntenic. This fact is contrasting with what is found in the ortologs from other organisms, like the human or mouse GLUT1, both as single-copy genes located in the chromosome 1 and 4, respectively, or Drosophilla melanogaster GLUT1, located in the chromosome $3 \mathrm{~L}$, in all cases flanked by 


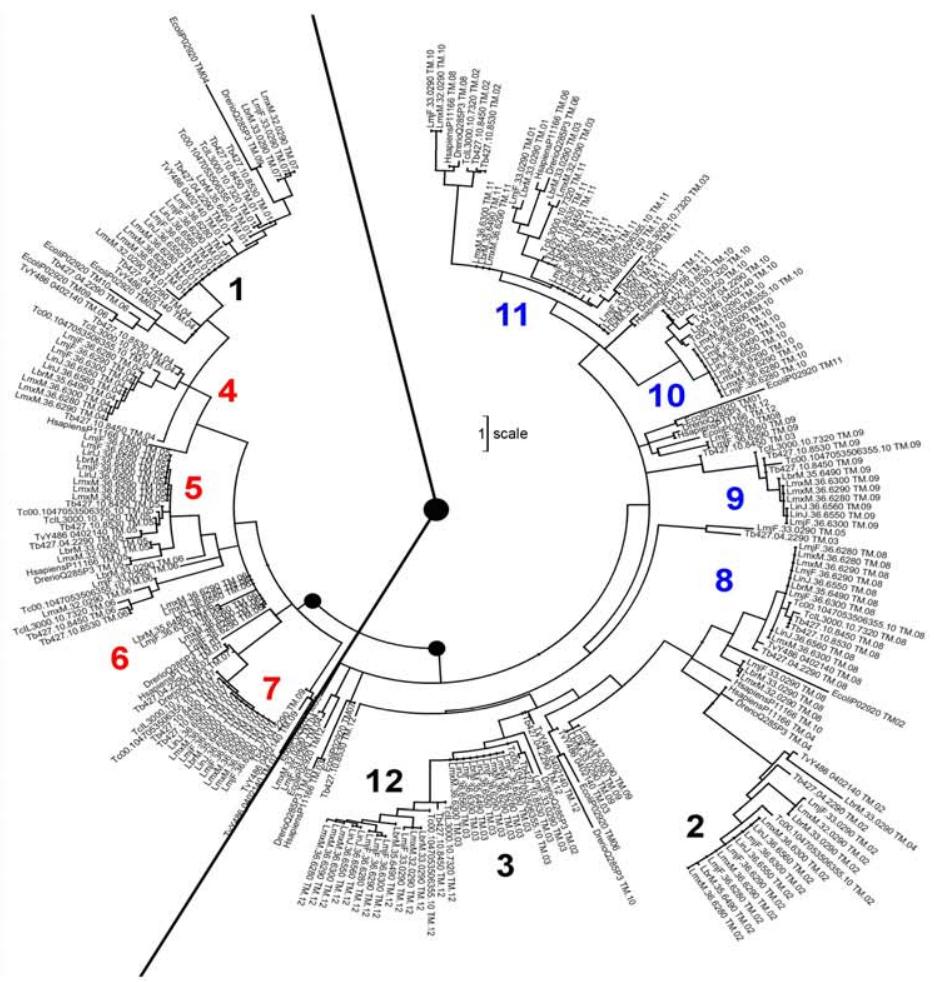

B
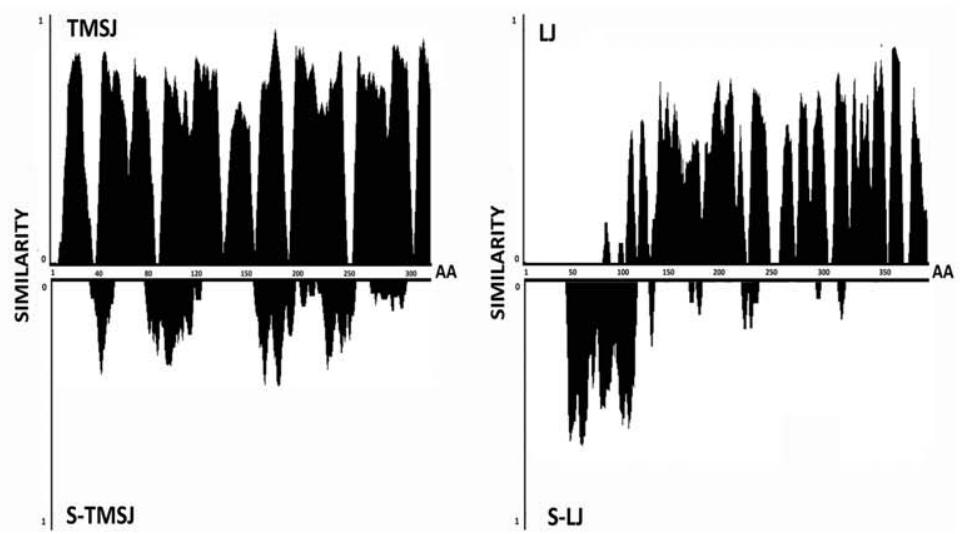

Figure 4. Evolution of each transmembrane spanindividually. A: Evolutionary tree using the pool of individual TMS sequences. A maximum likelihood method based on the JTT matrix-based model was used, with 500 bootstrap replicates. The tree is drawn to scale, with branch lengths measured in the number of substitutions per site. The tree with the highest log likelihood $(-3119.4504)$ is shown. Two major superclusters are separated by a solid line in which numbers indicate the main positions for the grouped TMSs. Colored numbers indicate consecutive TMSs from each supercluster. Scale bar corresponds to distance expressed in amino acid substitutions per site. B: Similarity as a function of position was plotted to compare TMSJ against S-TMSJ, and LJ against S-LJ.

doi:10.1371/journal.pone.0036303.g004

different pseudogenes. In synthesis, the genomic context is not conserved at all.

To summarize, as observed for the full-length sequences, phylogenetic analysis of the TMS, TMSJ and LJ sequences produced two major clusters that separated the sequences by genus. However, the phylogenetic trees constructed with the individual TMS sequences displayed the lowest mean distances, indicating that the TMSs are highly similar between proteins. This fact, together with the results obtained from the phylogenetic analysis, strongly supports the hypothesis that the loops are variable regions that are subject to the selective pressure of the immune system of the host. The TMSs, in contrast, are more constrained in terms of variation because they constitute the structural basis for the transport activity of the protein. The phylogenetic analysis of the pool of individual TMSs also showed their segregation in superclusters, some of which contain contiguous TMSs. This result indicates that the TMSs are positionally conserved and also suggests that the TMSs originated via intramolecular duplications. Nevertheless, the screening of the full-length sequences for internal repeats failed to detect consistently repeated sequences in the analyzed hexose transporters. The lack of data supporting duplications led us to conclude that the 
ancestral trypanosomatid already had a hexose transporter with multiple TMSs. However, we cannot rule out the occurrence of these kinds of events, which were reported as frequent in the original formation of the modules constituting these molecules.

\section{Supporting Information}

Figure S1 Evolutionary tree using the pool of individual L sequences. A maximum likelihood method based on the JTT matrix-based model was used, with 500 bootstrap replicates. The tree is drawn to scale, with branch lengths measured in the number of substitutions per site.

(DOC)

Figure S2 All 18 sequences were analyzed using the Multiple Em for Motif Elicitation (MEME) algorithm (http://meme.nbcr.net/). This analysis identified the motif QLTGINA (or the "GINA" motif), which was primarily present in TMS-6 and TMS-7. A. Sequence logo of the GINA motif. B. Conservation of the GINA motif in the analyzed sequences. C. Position of the GINA motif in the sequences.

(DOC)

Figure S3 The $T$. cruzi hexose transporter sequence was modeled based on the known structure of the Major Facilitator Superfamily (MFS) member LacY from $\boldsymbol{E}$. coli. Wire models of both transporters (T. cruzi: blue; E. coli LacY: yellow) are shown, with the T. cruzi structure overlaid on the E. coli structure. Both molecules appear to be heart shaped when represented in a view parallel to the membrane. The red arrow

\section{References}

1. Barrett MP, Burchmore RJ, Stich A, Lazzari JO, Frasch AC, et al. (2003) The trypanosomiases. Lancet 362: 1469-1480.

2. Silber AM, Colli W, Ulrich H, Alves MJ, Pereira CA (2005) Amino acid metabolic routes in Trypanosoma cruzi: possible therapeutic targets against Chagas' disease. Curr Drug Targets Infect Disord 5: 53-64.

3. Cazzulo JJ (1992) Aerobic fermentation of glucose by trypanosomatids. FASEB J 6: 3153-3161.

4. Tonelli RR, Silber AM, Almeida-de-Faria M, Hirata IY, Colli W, et al. (2004) $\mathrm{L}$-proline is essential for the intracellular differentiation of Trypanosoma cruzi. Cell Microbiol 6: 733-741.

5. Silber AM, Tonelli RR, Lopes CG, Gunha-e-Silva N, Torrecilhas AC, et al. (2009) Glucose uptake in the mammalian stages of Trypanosoma cruzi. Mol Biochem Parasitol 168: 102-108.

6. Cazzulo JJ (1994) Intermediate metabolism in Trypanosoma cruzi. J Bioenerg Biomembr 26: 157-165.

7. Bouvier LA, Silber AM, Galvao Lopes C, Canepa GE, Miranda MR, et al. (2004) Post genomic analysis of permeases from the amino acid/auxin family in protozoan parasites. Biochem Biophys Res Commun 321: 547-556.

8. Tetaud E, Barrett MP, Bringaud F, Baltz T (1997) Kinetoplastid glucose transporters. Biochem J 325 ( Pt 3): 569-580.

9. Barrett MP, Tetaud E, Seyfang A, Bringaud F, Baltz T (1998) Trypanosome glucose transporters. Mol Biochem Parasitol 91: 195-205.

10. Michels PA, Bringaud F, Herman M, Hannaert V (2006) Metabolic functions of glycosomes in trypanosomatids. Biochim Biophys Acta 1763: 1463-1477.

11. Landfear SM (2008) Drugs and transporters in kinetoplastid protozoa. Adv Exp Med Biol 625: 22-32.

12. El-Sayed NM, Myler PJ, Blandin G, Berriman M, Crabtree J, et al. (2005) Comparative genomics of trypanosomatid parasitic protozoa. Science 309: $404-409$.

13. Augustin R (2010) The protein family of glucose transport facilitators: It's not only about glucose after all. IUBMB Life 62: 315-333.

14. Henderson PJ, Maiden MC (1990) Homologous sugar transport proteins in Escherichia coli and their relatives in both prokaryotes and eukaryotes. Philos Trans R Soc Lond B Biol Sci 326: 391-410.

15. Hruz PW, Mueckler MM (2001) Structural analysis of the GLUT1 facilitative glucose transporter. Mol Membr Biol 18: 183-193.

16. Joost HG, Thorens B (2001) The extended GLUT-family of sugar/polyol transport facilitators: nomenclature, sequence characteristics, and potential function of its novel members. Mol Membr Biol 18: 247-256.

17. Aslett M, Aurrecoechea C, Berriman M, Brestelli J, Brunk BP, et al. (2010) TriTrypDB: a functional genomic resource for the Trypanosomatidae. Nucleic Acids Res 38: D457-462. indicates the direction of movement of the substrate (from the extracellular to the intracellular side).

(DOC)

Table S1 The number of amino acid substitutions per site between sequences is shown. Analyses were conducted using the JTT matrix-based model [21]. The analysis involved 216 amino acid sequences. All ambiguous positions were removed for each sequence pair. A total of 34 positions were included in the final dataset. Evolutionary analyses were conducted using MEGA5 [22].

(XLS)

\section{Dataset S1 Dataset of sequences in FASTA format.} (RAR)

\section{Acknowledgments}

Our special thanks to the presidents of Argentina and Brazil, Cristina Fernández de Kirchner and Dilma Rousseff, for their permanent policy of support of science in our countries.

\section{Author Contributions}

Conceived and designed the experiments: CAP AMS. Performed the experiments: CAP AMS. Analyzed the data: CAP AMS. Contributed reagents/materials/analysis tools: CAP AMS. Wrote the paper: CAP AMS. Conceived the initial hypothesis: CAP AMS. Designed and ran the bioinformatic work: CAP AMS. Data analysis, processed and interpreted the results: CAP AMS.

18. Hirokawa T, Boon-Chieng S, Mitaku S (1998) SOSUI: classification and secondary structure prediction system for membrane proteins. Bioinformatics 14: 378-379.

19. Krogh A, Larsson B, von Heijne G, Sonnhammer EL (2001) Predicting transmembrane protein topology with a hidden Markov model: application to complete genomes. J Mol Biol 305: 567-580.

20. Kumar S, Nei M, Dudley J, Tamura K (2008) MEGA: a biologist-centric software for evolutionary analysis of DNA and protein sequences. Brief Bioinform 9: 299-306.

21. Jones DT, Taylor WR, Thornton JM (1992) The rapid generation of mutation data matrices from protein sequences. Comput Appl Biosci 8: 275-282.

22. Tamura K, Peterson D, Peterson N, Stecher G, Nei M, et al. (2011) MEGA5: Molecular Evolutionary Genetics Analysis Using Maximum Likelihood, Evolutionary Distance, and Maximum Parsimony Methods. Mol Biol Evol.

23. Thompson JD, Higgins DG, Gibson TJ (1994) CLUSTAL W: improving the sensitivity of progressive multiple sequence alignment through sequence weighting, position-specific gap penalties and weight matrix choice. Nucleic Acids Res 22: 4673-4680.

24. Bailey TL, Elkan C (1994) Fitting a mixture model by expectation maximization to discover motifs in biopolymers. Proc Int Conf Intell Syst Mol Biol 2: 28-36.

25. Waitumbi JN, Tetaud E, Baltz T (1996) Glucose uptake in Trypanosoma vivax and molecular characterization of its transporter gene. Eur J Biochem 237: 234-239.

26. Vedrenne C, Bringaud F, Barrett MP, Tetaud E, Baltz T (2000) The structurefunction relationship of functionally distinct but structurally similar hexose transporters from Trypanosoma congolense. Eur J Biochem 267: 4850-4860.

27. Langford CK, Kavanaugh MP, Stenberg PE, Drew ME, Zhang W, et al. (1995) Functional expression and subcellular localization of a high- $\mathrm{Km}$ hexose transporter from Leishmania donovani. Biochemistry 34: 11814-11821.

28. Tetaud E, Bringaud F, Chabas S, Barrett MP, Baltz T (1994) Characterization of glucose transport and cloning of a hexose transporter gene in Trypanosoma cruzi. Proc Natl Acad Sci U S A 91: 8278-8282.

29. Tetaud E, Chabas S, Giroud C, Barrett MP, Baltz T (1996) Hexose uptake in Trypanosoma cruzi: structure-activity relationship between substrate and transporter. Biochem J 317 ( Pt 2): 353-359.

30. Rodriguez-Contreras D, Feng X, Keeney KM, Bouwer HG, Landfear SM (2007) Phenotypic characterization of a glucose transporter null mutant in Leishmania mexicana. Mol Biochem Parasitol 153: 9-18.

31. Burchmore RJ, Rodriguez-Contreras D, McBride K, Merkel P, Barrett MP, et al. (2003) Genetic characterization of glucose transporter function in Leishmania mexicana. Proc Natl Acad Sci U S A 100: 3901-3906.

32. Pao SS, Paulsen IT, Saier MH Jr. (1998) Major facilitator superfamily. Microbiol Mol Biol Rev 62: 1-34. 
33. Abramson J, Smirnova I, Kasho V, Verner G, Kaback HR, et al. (2003) Structure and mechanism of the lactose permease of Escherichia coli. Science 301: 610-615.

34. Guan L, Mirza O, Verner G, Iwata S, Kaback HR (2007) Structural determination of wild-type lactose permease. Proc Natl Acad Sci U S A 104: 15294-15298.

35. Landfear SM (2011) Nutrient transport and pathogenesis in selected parasitic protozoa. Eukaryot Cell 10: 483-493.

36. Barrett MP, Tetaud E, Seyfang A, Bringaud F, Baltz T (1995) Functional expression and characterization of the Trypanosoma brucei procyclic glucose transporter, THT2. Biochem J 312 ( Pt 3): 687-691.

37. Bringaud F, Baltz T (1994) African trypanosome glucose transporter genes: organization and evolution of a multigene family. Mol Biol Evol 11: 220-230.

38. Tetaud E, Bringaud F, Chabas S, Barrett MP, Baltz T (1994) Characterization of glucose transport and cloning of a hexose transporter gene in Trypanosoma cruzi. Proc Natl Acad Sci U S A 91: 8278-8282.
39. Tetaud E, Chabas S, Giroud C, Barrett MP, Baltz T (1996) Hexose uptake in Trypanosoma cruzi: structure-activity relationship between substrate and transporter. Biochem J 317 ( Pt 2): 353-359.

40. Burchmore RJ, Landfear SM (1998) Differential regulation of multiple glucose transporter genes in Leishmania mexicana. J Biol Chem 273: 29118-29126.

41. Krishna S, Woodrow CJ, Burchmore RJ, Saliba KJ, Kirk K (2000) Hexose transport in asexual stages of Plasmodium falciparum and kinetoplastidae. Parasitol Today 16: 516-521.

42. Mueckler M, Caruso C, Baldwin SA, Panico M, Blench I, et al. (1985) Sequence and structure of a human glucose transporter. Science 229: 941-945.

43. Hebert DN, Carruthers A (1992) Glucose transporter oligomeric structure determines transporter function. Reversible redox-dependent interconversions of tetrameric and dimeric GLUT1. J Biol Chem 267: 23829-23838.

44. Bunn RC, Jensen MA, Reed BC (1999) Protein interactions with the glucose transporter binding protein GLUT1CBP that provide a link between GLUT1 and the cytoskeleton. Mol Biol Cell 10: 819-832. 